\title{
Boundary value problems and $J$-complex curves
}

\author{
Alexandre Sukhov* and Alexander Tumanov**
}

\author{
* Université des Sciences et Technologies de Lille, Laboratoire Paul Painlevé, U.F.R. de Mathé- \\ matique, 59655 Villeneuve d'Ascq, Cedex, France, sukhov@math.univ-lille1.fr \\ ** University of Illinois, Department of Mathematics 1409 West Green Street, Urbana, IL 61801, \\ USA, tumanov@math.uiuc.edu
}

Abstract. We give a solution to the problem of filling by a Levi-flat hypersurface for a class of totally real tori in $\mathbb{C}^{2}$ equipped with a certain almost complex structure.

MSC: $32 \mathrm{H} 02,53 \mathrm{C} 15$.

Key words: almost complex structure, Levi-flat hypersurface, J-complex disc.

\section{Introduction}

The problem of gluing complex discs to real submanifolds in complex space has been a subject of extensive research. In this paper we consider the problem for a class of totally real tori in $\mathbb{C}^{2}$ equipped with a certain almost complex structure. We restrict to structures $J$ with the following characteristic property: the lines parallel to one coordinate axis are $J$-complex hypersurfaces. In complex dimension 2 every almost complex structure $J$ locally has this property; we impose this condition globally. We prove (Theorem 1.1) that certain real tori can be filled by Levi-flat hypersurfaces. The result can be viewed as a solution to a Riemann-Hilbert problem for a quasi-linear elliptic equation in the plane with non-linear boundary conditions. For the usual complex structure in $\mathbb{C}^{2}$, our result was obtained earlier by Forstnerič [6] and Schnirelman [11].

Let $(M, J)$ be an almost complex manifold. We denote by $\mathbb{D}$ the unit disc in $\mathbb{C}$. We denote by $J_{\text {st }}$ the standard complex structure of $\mathbb{C}^{n}$; the value of $n$ will be clear from context. A continuous map $f: \overline{\mathbb{D}} \rightarrow M$ differentiable in $\mathbb{D}$ is called a $J$-complex (or $J$-holomorphic) disc if it satisfies the equation $d f \circ J_{\text {st }}=J \circ d f$ in $\mathbb{D}$. We often identify a $J$-complex disc $f$ with the image $f(\overline{\mathbb{D}})$ and call it just a disc. By the boundary of the disc $f$ we mean the restriction $\left.f\right|_{b \mathbb{D}}$ which we also identify with its image.

In local coordinates $Z=(z, w) \in \mathbb{C}^{2}$, an almost complex structure $J$ can be defined by a complex matrix $A(Z)$ so that a map $Z: \mathbb{D} \rightarrow U$ is $J$-complex if and only if it satisfies the equation

$$
Z_{\bar{\zeta}}-A(Z) \overline{Z_{\zeta}}=0
$$


The matrix $A(Z)$ is defined by

$$
A(Z) v=\left(J_{\mathrm{st}}+J(Z)\right)^{-1}\left(J_{\mathrm{st}}-J(Z)\right) \bar{v} .
$$

Indeed, it is easy to see that the right-hand side of $(2)$ is $\mathbb{C}$-linear in $v \in \mathbb{C}^{2}$, hence it defines a unique matrix $A(Z)$ (see [13] for details). We call the matrix $A$ the complex matrix of $J$. The ellipticity of $(1)$ is equivalent to $\operatorname{det}(I-A \bar{A}) \neq 0$. In a fixed coordinate chart, the correspondence between almost complex structures $J$ with $\operatorname{det}\left(J_{\text {st }}+J\right) \neq 0$ and complex matrices with $\operatorname{det}(I-A \bar{A}) \neq 0$ is one-to-one [13].

Let $A$ be a lower triangular complex matrix function in a domain in $\mathbb{C}^{2}$. That is,

$$
A=\left(\begin{array}{ll}
a & 0 \\
b & c
\end{array}\right)
$$

The matrix $A$ is the matrix of an almost complex structure if and only if $|a| \neq 1$ and $|c| \neq 1$. An almost complex structure $J$ with matrix $A$ of the form (3) has the following characteristic property: the lines $z=$ const are $J$-complex curves, that is, their tangent spaces are $J$-invariant.

Our main result is the following

Theorem 1.1 Let $J$ be a smooth almost complex structure on $\overline{\mathbb{D}} \times \mathbb{C}$ with matrix (3), in which $|a|<a_{0},|c|<a_{0}$, and $0<a_{0}<1$ is a constant. Suppose the map $z \mapsto(z, 0)$ is $J$-complex, that is, $a(z, 0)=b(z, 0)=0$. Suppose the first derivatives of $a, b$, and $c$ with respect to $w$ and $\bar{w}$ are uniformly bounded. Let $\gamma_{z} \subset \mathbb{C}$ be a smooth simple closed curve depending smoothly on the parameter $z \in b \mathbb{D}$. Suppose that for every $z \in b \mathbb{D}$, the bounded component of $\mathbb{C} \backslash \gamma_{z}$ contains 0 . Introduce the torus $\Lambda=\bigcup_{z \in b \mathbb{D}}\{z\} \times \gamma_{z}$. Then the following hold.

(i) For every point $p \in \Lambda$ there exists a J-complex disc $f: \overline{\mathbb{D}} \rightarrow \overline{\mathbb{D}} \times \mathbb{C}$ such that $f(1)=p$ and whose image $f(\overline{\mathbb{D}})$ is a graph of a non-vanishing function $z \mapsto w(z)$ of class $C^{\infty}(\overline{\mathbb{D}})$, that is, $f(\overline{\mathbb{D}})=\{(z, w): w=w(z), z \in \overline{\mathbb{D}}\}$. In particular, $f$ is an embedding, $f(b \mathbb{D}) \subset \Lambda$, and $f(\overline{\mathbb{D}})$ does not meet $\overline{\mathbb{D}} \times\{0\}$. The disc $f$ is unique up to parametrization in any Sobolev class $W^{1, p}(\mathbb{D}), p>2$.

(ii) The discs in (i) form a $C^{\infty}$-smooth one-parameter family; they depend continuously on the matrix $A$ and the curves $\gamma_{z}$. They are disjoint and fill a smooth Levi-flat hypersurface $\Gamma \subset \overline{\mathbb{D}} \times \mathbb{C}$ with boundary $\Lambda$.

(iii) There is a constant $C>0$ that depends only on $A$ such that if $\gamma_{z} \subset r \overline{\mathbb{D}}$ for some $r>0$ and all $z \in b \mathrm{D}$, then $\Gamma \subset \overline{\mathrm{D}} \times R \overline{\mathrm{D}}, R=C r$.

The hypothesis that the matrix $A$ has a special form (3) is natural because it is tied to the special form of the domain $\overline{\mathbb{D}} \times \mathbb{C}$. In particular, it guarantees that the torus $\Lambda$ is totally real. For a general matrix $A$ the conclusion of the theorem fails. 
As we mention above, in the integrable case $(A=0)$, this result was obtained earlier by Forstnerič [6] and Schnirelman [11]. Already in this special case, Theorem 1.1 has important connections. Berndtsson and Ransford [1] used a suitable version in their proof of the corona theorem. Slodkowski [12] used similar ideas in his $\lambda$-lemma, which has important applications in complex dynamics and quasi-conformal maps. Recently Duval and Gayet [5] obtained a new result on gluing holomorphic discs and annuli to certain totally real tori in $\mathbb{C}^{2}$. In the integrable case, Theorem 1.1 can be used to study envelopes of holomorphy following Bedford and Klingenberg [2] and Forstnerič [6]. Another direction concerns applications in symplecic and contact geometry in the spirit of works of Gromov [7], Eliashberg [8], and others. Here Theorem 1.1 may be used in full generality, that is, for non-integrable almost complex structures. In a forthcoming paper we will use Theorem 1.1 for describing deformations of $J$-complex discs. Finally, a situation related to that in Theorem 1.1 often arises in the theory of holomorphic foliations, see for instance [3]. We hope that our result will find further applications in these directions and will be a useful tool in their development.

\section{Elliptic estimates and the maximum principle}

We first consider a non-homogeneous Beltrami equation

$$
v_{\bar{z}}=q v_{z}+Q
$$

The following result must be well known, however we could not find a precise reference. For completeness we include a proof.

Proposition 2.1 (i) Let $q$ and $Q$ be bounded functions in $\mathbb{D},|q| \leq q_{0}<1,|Q| \leq Q_{0}$, here $q_{0}$ and $Q_{0}$ are constants. There exists $p>2$ and a unique solution $v$ of (4) in the Sobolev class $W^{1, p}(\mathbb{D})$ with boundary conditions $\left.\operatorname{Re} v\right|_{b \mathbb{D}}=0, v(1)=0$. Furthermore $\|v\|_{C^{\alpha}(\overline{\mathbb{D}})} \leq C$ with $\alpha=\frac{p-2}{p}$. Here $C>0$ and $p$, hence $\alpha$, depend on $q_{0}$ and $Q_{0}$ only.

(ii) If in addition $\|q\|_{C^{k, \beta}(\overline{\mathbb{D}})}+\|Q\|_{C^{k, \beta}(\overline{\mathrm{D}})} \leq Q_{0}$ for some $0<\beta<1$ and $k \geq 0$, then $\|v\|_{C^{k+1, \beta}(\overline{\mathbb{D}})} \leq C$; here $C>0$ depends on $\beta, k, q_{0}$ and $Q_{0}$ only.

The statement (ii) is not needed in this paper. We include it for completeness and future references.

Proof : (i) We use a classical method for solving the Beltrami equation based on the Cauchy-Green operator $T$ and its modification ([14], Theorem 3.29; see also [4], equation $(5))$ :

$$
T u(z)=\frac{1}{2 \pi i} \int_{\mathbb{D}} \frac{u(\zeta) d \zeta \wedge d \bar{\zeta}}{\zeta-z}, \quad T_{1} u(z)=T u(z)-\overline{T u\left(z^{*}\right)}-2 i \operatorname{Im} T u(1)
$$

here $z^{*}:=1 / \bar{z}$. Then $v=T_{1} u$ solves the boundary value problem: $v_{\bar{z}}=u,\left.\operatorname{Re} v\right|_{b \mathbb{D}}=0$, $v(1)=0$. We also use $S_{1} u(z)=\partial_{z} T_{1} u(z)$. The operator $S_{1}$ is an isometry of $L^{2}(\mathbb{D})$. 
Put $u:=v_{\bar{z}}$. Then we have $v=T_{1} u$ and $v_{z}=S_{1} u$. Then (4) turns into

$$
u=q S_{1} u+Q .
$$

Since $|q| \leq q_{0}<1$ and $\left\|S_{1}\right\|_{L^{2}}=1$, then for $p>2$ close to 2 , we have $\left\|q S_{1}\right\|_{L^{p}}<1$. Hence (5) has a unique solution $u \in L^{p}(\mathbb{D})$. Then $v=T_{1} u \in W^{1, p}(\mathbb{D})$. Then $v \in C^{\alpha}, \alpha=\frac{p-2}{p}$. The solution is unique in $W^{1, p}$ and its $C^{\alpha}$ norm is estimated in terms of $q_{0}$ and $Q_{0}$ only.

(ii) Let $\xi: \overline{\mathbb{D}} \rightarrow \overline{\mathrm{D}}$ be a Beltrami homeomorphism satisfying the equation

$$
\xi_{\bar{z}}=q \xi_{z}, \quad \xi(1)=1 .
$$

Since $q \in C^{k, \beta}$, then $\xi \in C^{k+1, \beta}$ is a diffeomorphism (see [14], Theorem 2.10). By changing the independent variable to $\xi$, the equation (4) turns into

$$
v_{\bar{\xi}} \bar{\xi}_{\bar{z}}\left(1-|q|^{2}\right)=Q .
$$

Since $\xi \in C^{k+1, \beta}(\mathbb{D})$, then the change of variable $z \rightarrow \xi$ preserves the class $C^{k, \beta}(\mathbb{D})$. Hence

$$
v=T_{1} \frac{Q}{\bar{\xi}_{\bar{z}}\left(1-|q|^{2}\right)},
$$

here $T_{1}$ is applied with respect to the variable $\xi$. It shows that $v \in C^{k+1, \beta}(\mathbb{D})$. It is clear from the construction that the norm of $v$ is estimated in terms of $\beta, q_{0}$, and $Q_{0}$ only. The proof is complete. Q.E.D.

We consider some properties of almost complex structures with matrices of the form (3). We begin with coordinate changes that preserve this form.

Proposition 2.2 Let $A$ be a complex matrix of a smooth almost complex structure $J$ in a domain in $\mathbb{C}^{2}$ with coordinates $Z=(z, w)$. Suppose $A$ has the form (3) with $|a|<1$ and $|c|<1$. Let $Z^{\prime}=\left(z^{\prime}, w^{\prime}\right)$ be a smooth orientation preserving coordinate change of the form $z^{\prime}=z, w^{\prime}=w^{\prime}(z, w)$. Then the matrix $A^{\prime}$ of $J$ relative to the new coordinates also has the form (3). Furthermore, if $w(z, 0)=0, a(z, 0)=0$, and $b(z, 0)=0$, then for the corresponding entries of $A^{\prime}$ we have $a^{\prime}\left(z^{\prime}, 0\right)=0$ and $b^{\prime}\left(z^{\prime}, 0\right)=0$. (Finally, if in addition $w_{\bar{w}}^{\prime}(z, 0)=0$ and $c(z, 0)=0$, then $c^{\prime}\left(z^{\prime}, 0\right)=0$.)

Proof : We represent the coordinate change in an equivalent form $z=z^{\prime}, w=w\left(z^{\prime}, w^{\prime}\right)$. According to [13], the matrix $A$ changes to

$$
A^{\prime}=\left(Z_{Z^{\prime}}-A \bar{Z}_{Z^{\prime}}\right)^{-1}\left(A \bar{Z}_{\bar{Z}^{\prime}}-Z_{\bar{Z}^{\prime}}\right) .
$$

Then the matrix $A^{\prime}$ has the form (3) with entries

$$
\begin{aligned}
& a^{\prime}=a, \\
& b^{\prime}=\left(w_{w^{\prime}}-c \bar{w}_{w^{\prime}}\right)^{-1}\left(b-a\left(w_{z^{\prime}}-c \bar{w}_{z^{\prime}}\right)+c \bar{w}_{\bar{z}^{\prime}}-w_{\bar{z}^{\prime}}\right), \\
& c^{\prime}=\left(w_{w^{\prime}}-c \bar{w}_{w^{\prime}}\right)^{-1}\left(c \bar{w}_{\bar{w}^{\prime}}-w_{\bar{w}^{\prime}}\right) .
\end{aligned}
$$


Note that $w_{w^{\prime}}-c \bar{w}_{w^{\prime}} \neq 0$ because the change or variables is orientation preserving and $|c|<1$. The conclusions now follow from the above expressions of $a^{\prime}, b^{\prime}$, and $c^{\prime}$. Q.E.D.

For almost complex structures with matrix (3), one can reduce the Cauchy-Riemann system to a single equation.

Proposition 2.3 Let $\zeta \rightarrow(z(\zeta), w(\zeta))$ be a J-complex curve for an almost complex structure with matrix (3), where $|a|<1$ and $|c|<1$. Suppose $z_{\zeta} \neq 0$. Then the map locally can be represented by a graph of a function $z \mapsto w(z)$ satisfying the equation

$$
w_{\bar{z}}=a_{1} w_{z}+c_{1} \bar{w}_{\bar{z}}+b_{1}
$$

whose coefficients are determined by $A$. The equation is elliptic, in particular $\left|a_{1}\right|+\left|c_{1}\right|<1$. Moreover, if $a(z, 0)=0$ (resp. $b(z, 0)=0, c(z, 0)=0)$, then $a_{1}(z, 0)=0\left(\right.$ resp. $b_{1}(z, 0)=0$, $\left.c_{1}(z, 0)=0\right)$. Conversely, if $z \mapsto w(z)$ satisfies (6), then its graph locally can be represented as a parametrized $J$-complex curve. Finally, the correspondence between the triples $(a, b, c)$ with $|a|<1$ and $|c|<1$ and the triples $\left(a_{1}, b_{1}, c_{1}\right)$ with $\left|a_{1}\right|+\left|c_{1}\right|<1$ is one-to-one.

Proof : By the Cauchy-Riemann equations (1),

$$
z_{\bar{\zeta}}=a \bar{z}_{\bar{\zeta}}, \quad w_{\bar{\zeta}}=b \bar{z}_{\bar{\zeta}}+c \bar{w}_{\bar{\zeta}}
$$

By the Chain Rule,

$$
w_{\zeta}=w_{z} z_{\zeta}+w_{\bar{z}} \bar{z}_{\zeta}, \quad w_{\bar{\zeta}}=w_{z} z_{\bar{\zeta}}+w_{\bar{z}} \bar{z}_{\bar{\zeta}}
$$

By eliminating $z_{\bar{\zeta}}, w_{\zeta}$, and $w_{\bar{\zeta}}$, and canceling by $\bar{z}_{\bar{\zeta}} \neq 0$ we obtain

$$
w_{\bar{z}}-a c \bar{w}_{z}=-a w_{z}+c \bar{w}_{\bar{z}}+b .
$$

Plugging (6) in (8) yields

$$
a_{1}-a c \bar{c}_{1}=-a, \quad b_{1}-a c \bar{b}_{1}=b, \quad c_{1}-a c \bar{a}_{1}=c .
$$

Solving (9) yields the coefficients of the desired equation (6):

$$
a_{1}=-a\left(1-|c|^{2}\right) / \Delta, \quad b_{1}=(b+a c \bar{b}) / \Delta, \quad c_{1}=c\left(1-|a|^{2}\right) / \Delta, \quad \Delta=1-|a c|^{2},
$$

in which $\left|a_{1}\right|+\left|c_{1}\right|<1$. Conversely, if $w(z)$ satisfies (6), then we can find the parameter $\zeta(z)$ by solving the linear Beltrami equation $\zeta_{\bar{z}}+a(z, w(z)) \zeta_{z}=0$, which is the first equation in (7) written for the inverse function $z \mapsto \zeta(z)$.

Finally, we observe that the equations (9) have a unique solution in $(a, b, c)$ with $|a|<$ $1,|c|<1$. Indeed, put $q=a c$. Then (9) imply $\left(a_{1}-q \bar{c}_{1}\right)\left(c_{1}-q \bar{a}_{1}\right)=-q$. The latter has only one solution $q$ with $|q|<1$ because $\left|a_{1}\right|+\left|c_{1}\right|<1$. The equations (9) now give the triple $(a, b, c)$ with $|a|<1,|c|<1$. The other conclusions follow automatically. Q.E.D.

We need a maximum principle for solutions of (6). It is mentioned in [14] without proof. For completeness we include it here. Consider a linear equation

$$
w_{\bar{z}}=q_{1} w_{z}+q_{2} \bar{w}_{\bar{z}}+Q_{1} w+Q_{2} \bar{w}
$$


Proposition 2.4 Let $q_{1}, q_{2}, Q_{1}, Q_{2}$ be bounded functions in $\overline{\mathbb{D}}$ and let $\left|q_{1}\right|+\left|q_{2}\right| \leq q_{0}<1$, $\left|Q_{1}\right|+\left|Q_{2}\right| \leq Q_{0}$, here $q_{0}$ and $Q_{0}$ are constants.

(i) There exists a constant $C>0$ depending only on $q_{0}$ and $Q_{0}$ so that for solutions of the equation (11) we have $\max _{\mathbb{D}}|w| \leq C \max _{b \mathbb{D}}|w|$.

(ii) If a solution to the equation (11) satisfies $\left.\operatorname{Re} w\right|_{b \mathbb{D}}=0$, then either $w$ does not vanish in $\overline{\mathbb{D}}$ or $w \equiv 0$.

We note that $\left\|Q_{1}\right\|_{L^{p}}+\left\|Q_{2}\right\|_{L^{p}} \leq Q_{0}$ for some $p>2$ instead of $p=\infty$ would suffice, but we do not need it here.

Proof : $\quad$ Let $w$ be a solution of (11). Put $q=q_{1}+q_{2} \frac{\bar{w}_{\bar{z}}}{w_{z}}$ and $Q=Q_{1}+Q_{2} \frac{\bar{w}}{w}$. Then $w$ also satisfies

$$
w_{\bar{z}}=q w_{z}+Q w .
$$

Let $u$ and $v$ be solutions of the Beltrami equations

$$
u_{\bar{z}}=q u_{z}, \quad v_{\bar{z}}=q v_{z}+Q
$$

so that $u: \overline{\mathbb{D}} \rightarrow \overline{\mathbb{D}}$ is a homeomorphism. Such solutions exist because $|q| \leq q_{0}<1$. Then one can see ([14], Theorem 3.31) that every solution of (12), whence (11), has a representation

$$
w(z)=\phi(u(z)) e^{v(z)},
$$

where $\phi$ is holomorphic in $\mathbb{D}$. Indeed, define $\phi$ by (14) and plug in (12). Then using (13) we obtain $\left(1-|q|^{2}\right) \bar{u}_{\bar{z}} e^{v} \phi_{\bar{u}}=0$. Hence $\phi_{\bar{u}}=0$, and $\phi$ is holomorphic.

Although the equations (13) depend on a solution $w$, by Proposition 2.1(i) there exists $v$ whose sup-norm depends on $q_{0}$ and $Q_{0}$ only. Hence, the assertion (i) follows by the usual maximum principle applied to a holomorphic function $\phi$.

To prove (ii), we note that by Proposition 2.1(i), the solution $v$ to the non-homogeneous equation in (13) can be chosen with the condition $\left.\operatorname{Im} v\right|_{b \mathbb{D}}=0$. Then $\left.\operatorname{Re} w\right|_{b \mathbb{D}}=0$ implies $\left.\operatorname{Re} \phi\right|_{b \mathbb{D}}=0$. Since $\phi$ is holomorphic, then $\phi \equiv i c$, where $c \in \mathbb{R}$ is constant, and (ii) follows. The proof is complete. Q.E.D.

Corollary 2.5 Let the coefficients $a_{1}$ and $c_{1}$ of (6) satisfy $\left|a_{1}\right|+\left|c_{1}\right| \leq a_{0}<1$ and let $\left|\left(b_{1}\right)_{w}\right|+\left|\left(b_{1}\right)_{\bar{w}}\right| \leq b_{0}$; here $a_{0}$ and $b_{0}$ are constants. Suppose $b_{1}(z, 0)=0$. Then there exists a constant $C>0$ depending only on $a_{0}$ and $b_{0}$ so that for every solution of (6) we have $\max _{\mathbb{D}}|w| \leq C \max _{b \mathbb{D}}|w|$.

Proof : Our hypotheses on $b_{1}$ imply that $b_{1}(z, w)=Q_{1}(z, w) w+Q_{2}(z, w) \bar{w}$, where $\left|Q_{1}\right| \leq b_{0}$ and $\left|Q_{2}\right| \leq b_{0}$. Then the conclusion follows by Proposition 2.4(i). Q.E.D.

Finally we include a result on the regularity of the boundary value problem for (6).

Proposition 2.6 Let $w \in W^{1, p}(\mathbb{D}), p>2$, be a solution of (6) satisfying the boundary condition $\left.\operatorname{Re} w\right|_{b \mathbb{D}}=\phi$. Suppose that $\left|a_{1}\right|+\left|c_{1}\right|<1, a_{1}, b_{1}, c_{1} \in C^{k, \alpha}(\mathbb{D} \times \mathbb{C}), 0<\alpha<1$, and $\phi \in C^{k+1, \alpha}(\mathbb{D}), k \geq 0$. Then $w \in C^{k+1, \alpha}(\mathbb{D})$. 
Proof : We interpret the graphs of solutions of (6) as $J$-complex discs and apply a reflection principle from [9]. According to it, if $J$ is $C^{k, \alpha}(k \geq 0,0<\alpha<1)$, then $J$-complex discs attached to a totally real submanifold are $C^{k+1, \alpha}$-smooth.

Let $J$ be the almost complex structure corresponding to the equation (6) by Proposition 2.3. Then the solution of (6) with boundary condition $\left.\operatorname{Re} w\right|_{b \mathbb{D}}=\phi$ defines a $J$-complex disc attached to the totally real submanifold $\{(z, w):|z|=1$, Re $w=\phi(z)\}$. The conclusion now follows by the reflection principle [9]. Q.E.D.

\section{Proof of the main theorem}

Without loss of generality we can assume $\Lambda=b \mathrm{D} \times b \mathbb{D}$. Indeed, otherwise we can transform the curves $\{z\} \times \gamma_{z}$ into the circles $\{z\} \times b \mathbb{D}$ by a smooth change of coordinates of the form $z^{\prime}=z, w^{\prime}=w^{\prime}(z, w)$ so that $w^{\prime}(z, 0)=0$ and $w^{\prime}=w$ for big $w$. By Proposition 2.2, the hypotheses of the theorem will be preserved by the change.

By Proposition 2.3, if a smooth $J$-complex disc in $\overline{\mathbb{D}} \times \mathbb{C}$ can be represented as a graph of a smooth function $w: \overline{\mathbb{D}} \rightarrow \mathbb{C}$, then $w$ satisfies the equation (6). The disc will be attached to $\Lambda=b \mathbb{D} \times b \mathbb{D}$ if in addition $|w(z)|=1$ for $z \in b \mathbb{D}$.

Without loss of generality we can assume that the coefficients $a, b$, and $c$ have compact support in $\overline{\mathbb{D}} \times \mathbb{C}$. Indeed, by the maximum principle (Corollary 2.5 ), the boundary condition $|w|=1$ implies $|w|<C$ for all $z \in \mathbb{D}$. Here $C>0$ depends only on the coefficients of (6). Then we can assume $a=b=c=0$, hence $a_{1}=b_{1}=c_{1}=0$ for $|w|>C$.

Since we are looking for a non-vanishing solution $w$, we now make a change of variable $w=e^{u}$. Then the equation (6) with boundary condition $|w|=1$ transforms into

$$
u_{\bar{z}}=a_{2} u_{z}+c_{2} \bar{u}_{\bar{z}}+b_{2}
$$

with boundary condition

$$
\operatorname{Re} u(z)=0, z \in b \mathbb{D} \text {. }
$$

Here

$$
a_{2}(z, u)=a_{1}\left(z, e^{u}\right), \quad b_{2}(z, u)=e^{-u} b_{1}\left(z, e^{u}\right), \quad c_{2}(z, u)=e^{\bar{u}-u} c_{1}\left(z, e^{u}\right) .
$$

The coefficients of (15) still have uniformly bounded derivatives in $u$ and satisfy the ellipticity condition $\left|a_{2}\right|+\left|c_{2}\right|<a_{0}<1$. Under these hypotheses by [10] (pp. 335-351; see also [13], Proposition 4.2) for every every $z_{0} \in b \mathbb{D}$ and $\tau \in \mathbb{R}$, the boundary value problem (15-16) has a unique solution with $u\left(z_{0}\right)=i \tau$.

Returning to the original variable $w$, we obtain a one-parameter family of discs attached to $\Lambda$. They are parametrized by $\tau \in \mathbb{R} / 2 \pi \mathbb{Z}$ for fixed $z_{0}$, say $z_{0}=1$. Their boundaries are disjoint and cover all of $\Lambda$. Since $w=e^{u} \neq 0$, then they do not meet $\overline{\mathbb{D}} \times\{0\}$. By the continuous dependence statement of [10], the family is continuous. For the same reason, they depend continuously on $A$, which gives the continuous dependence conclusion in (ii).

The smoothness of the above one-parameter family follows by the implicit function theorem. Fix $k \geq 1,0<\alpha<1$. By Proposition 2.6, every solution $u \in C^{k+1, \alpha}(\mathbb{D})$. Denote by 
$L: C^{k, \alpha}(\mathbb{D}) \rightarrow C^{k-1, \alpha}(\mathbb{D}), L: \dot{u} \mapsto L(\dot{u})$, the linearization at $u$ of the operator defined by (15). Then the operator $L$ itself can be written in the same form with coefficients of class $C^{k-1, \alpha}(\mathbb{D})$. By Proposition 2.6 the linear map $\mathcal{L}: C^{k, \alpha}(\mathbb{D}) \rightarrow C^{k-1, \alpha}(\mathbb{D}) \times C^{k, \alpha}(b \mathbb{D})$ defined by $\mathcal{L}(\dot{u})=\left(L(\dot{u}),\left.\operatorname{Re} \dot{u}\right|_{b \mathbb{D}}\right)$ is surjective. As it was shown above, the solution of the boundary value problem (15-16) is uniquely determined by the condition $u\left(z_{0}\right)=i \tau$ for every $z_{0} \in b \mathbb{D}$ and $\tau \in \mathbb{R}$. Hence the kernel of $\mathcal{L}$ is one-dimensional. By the implicit function theorem there exists a $C^{k, \alpha}$-smooth one-parameter family of solutions of (15-16). By uniqueness we obtain the smooth dependence conclusion in (ii).

The fact that the discs are disjoint follows by the positivity of intersections of $J$-complex curves. Indeed, we can include our boundary value problem $|w|=1$ for the equation (6) in a one-parameter family with boundary condition $|w|=r$. Let $f$ be a $J$-complex disc constructed above, attached to $\Lambda$. Then we can construct a continuous family of $J$-complex discs $f_{r}$ with boundary condition $|w|=r$, so that $f_{0}$ is the disc $\overline{\mathbb{D}} \times\{0\}$ and $f_{1}=f$. Let $g$ be another disc constructed above, attached to $\Lambda$. Since the boundaries of $g$ and $f_{r}$ do not intersect, then the intersection index of $f_{r}$ and $g$ is independent of $r$. Since $g$ does not intersect $f_{0}$, then it does not intersect $f_{1}=f$ either.

We now prove that the surface $\Gamma$ swept out by the discs is smooth. Let $z \mapsto u(z, \tau)$ be the solution of $(15)$ with conditions $\left.\operatorname{Re} u\right|_{b \mathbb{D}}=0$ and $u(1, \tau)=i \tau$. It suffices to show that the map $(z, \tau) \mapsto(z, u(z, \tau))$ is an immersion, which in turn reduces to $\partial u / \partial \tau \neq 0$. Put $v=\partial u / \partial \tau$ and differentiate (15) with respect to $\tau$. Then we obtain a linear equation

$$
v_{\bar{z}}=a_{2} v_{z}+c_{2} \bar{v}_{\bar{z}}+Q_{1} v+Q_{2} \bar{v}
$$

with boundary condition $\left.\operatorname{Re} v\right|_{b \mathbb{D}}=0$. Here $Q_{1}=\left(a_{2}\right)_{u} u_{z}+\left(c_{2}\right)_{u} \bar{u}_{\bar{z}}+\left(b_{2}\right)_{u}$ and $Q_{2}=$ $\left(a_{2}\right)_{\bar{u}} u_{z}+\left(c_{2}\right) \bar{u}_{\bar{u}}+\left(b_{2}\right)_{\bar{u}}$. Since $v(1)=i \neq 0$, then by Proposition $2.4(\mathrm{ii})$, the solution $v$ does not vanish in $\overline{\mathrm{D}}$ as desired.

Finally, the part (iii) follows by Corollary 2.5, the maximum principle for (6). The proof is complete. Q.E.D.

\section{References}

[1] B. Berndtsson and T. Ransford, Analytic multifunctions, the $\bar{\partial}$-equation, and a proof of the corona theorem, Pacific J. Math. 124 (1986), 57-72.

[2] E. Bedford and W. Klingenberg, Envelopes of holomorphy of certain 2-spheres in $\mathbb{C}^{2}$, J. Amer. Math. Soc. 4 (1991), 623-646.

[3] M. Brunella, Subharmonic variation ofthe leafwise Poincaré metric, Invent. Math. 152 (2003), 119148.

[4] B. Coupet, A. Sukhov, and A. Tumanov, Proper J-holomorphic discs in Stein domains of dimension 2, Amer. J. Math. 131 (2009), 653-674.

[5] J. Duval and D. Gayet, Riemann surfaces and totally real tori, arXiv 0910.2139.

[6] F. Forstnerič, Polynomial hulls of sets fibered over the unit circle, Indiana Univ. Math. J. 37(1988), 869-889. 
[7] M. Gromov, Pseudoholomorphic curves in symplecic manifolds, Invent. Math. 82 (1985), 307-347.

[8] Y. Eliashberg, Filling by holomorphic discs and its applications, Geometry of low-dimensional manifolds, 2 (Durham, 1989), 45-67, London Math. Soc. Lecture Series, 151, Cambridge Univ. Press, Cambridge, 1990.

[9] S. Ivashkovich and A. Sukhov, Schwarz reflection principle, boundary regularity and compactness for $J$-complex curves, Ann. Inst. Fourier (Grenoble) 60 (2010), 1489-1513.

[10] V. N. Monakhov, Boundary problems with free boundary for elliptic systems of equations, Translations of Mathematical Monographs, 57, Amer. Math. Soc., Providence, 1983, 522 pp.

[11] A. Schnirelman, Degree of a quasilinear map and the non-linear Riemann-Hilbert problem (Russian), Matem. Sb. 89 (1972), 366-389.

[12] Z. Slodkowski, Holomorphic motions and polynomial hulls, Proc. Amer. Math.Soc. 111 (1991), 347355 .

[13] A. Sukhov and A. Tumanov, Regularization of almost complex structures and constructions of pseudoholomorphic discs, arXiv 0809.4651, to appear in Annali Scuola Norm. Sup. Pisa.

[14] I. N. Vekua, Generalized analytic functions, Pergamon, London-Paris-Frankfurt, 1962. 